\title{
Dissecting anxiety-related QTLs in mice by univariate and multivariate mapping
}

\author{
ZHU ZhiHong ${ }^{1}$, ZHANG ChenHao ${ }^{1}$, WANG XuSheng ${ }^{3}$, COOK Melloni ${ }^{4}$, \\ WILLIAMS Robert ${ }^{3}, \mathrm{LU} \mathrm{Lu}^{2,3^{*}} \& \mathrm{ZHU}^{\mathrm{J}} \mathrm{Ju}^{1 *}$ \\ ${ }^{1}$ ZJU-IBM Biocomputation Laboratory, College of Agriculture and Biotechnology, Zhejiang University, Hangzhou 310058, China; \\ ${ }^{2}$ Jiangsu Key Laboratory of Neuroregeneration, Nantong University, Nantong 226001, China; \\ ${ }^{3}$ Department of Anatomy and Neurobiology, University of Tennessee Healthy Science Center, Memphis, TN 38163, USA; \\ ${ }^{4}$ Department of Psychology, University of Memphis, Memphis, TN 38152, USA
}

Received March 19, 2012; accepted April 23, 2012

\begin{abstract}
To detect genes underlying anxiety-related traits in mice, we performed univariate and multivariate QTL mapping analyses of phenotypes obtained from 71 mice of the BXD recombinant inbred (RI) strains ( $n=528$ mice) and their parental strains (C57BL/6J and DBA/2J). Separate and joint mapping analyses were carried out using a linkage map composed of 506 simple sequence repeats (SSRs). The main QTL effects, interactions between pairs of QTLs (epistasis), and their environmental interactions were estimated. The results showed that anxiety-related traits were influenced by multiple QTLs (five main effect QTLs and three epistatic QTLs). Ten potential anxiety-related candidate genes within the QTL intervals on chromosomes 5, 13 and 15 were identified. Some of these genes have been reported previously to be associated with the anxiety response. Based on our results, it is suggested that the multivariate QTL mapping approach improves the statistical power for detecting QTL and the precision of parameter estimation. Moreover, multivariate mapping can also detect pleiotropic QTL effects.
\end{abstract}

multivariate QTL mapping, mouse, BXD strains, pleiotropy

Citation: $\quad$ Zhu Z H, Zhang C H, Wang X S, et al. Dissecting anxiety-related QTLs in mice by univariate and multivariate mapping. Chin Sci Bull, 2012, 57: 2727-2732, doi: 10.1007/s11434-012-5240-x

Complex traits, including many common diseases, are controlled by multiple loci, each of which can be detected as a quantitative trait locus (QTL). Most approaches map QTLs separately by a phenotype-driven method focusing on an individual trait [1]. However, complex trait data usually contain observations on multiple traits either in multiple environments or under different treatment conditions. Therefore, several potential problems arise; for instance, determining whether multiple traits are affected by a single QTL with pleiotropic effects or by multiple closely linked QTLs. Additionally, the effects of QTLs may vary in different environments or under different treatment conditions. Several studies have suggested that genotype-environment interac-

*Corresponding authors (email: jzhu@zju.edu.cn; lulu521uthsc@gmail.com) tions $(G \times E)$ play important roles in gene-associated diseases, and thus can be thought of as a potential factor underlying the genetic variation of quantitative traits [2-4].

Recombinant inbred (RI) strains are an important resource that has been used to map complex traits. They provide compelling advantages for mapping complex traits. In mice, the largest RI set, the BXD strains, has been extensively used for mapping traits with complex genetic and environmental effects, including mood and anxiety disorders [5-8]. Previous studies have shown that the BXD RI strains have marked variation in anxiety- and fear-related phenotypes. Differences in these phenotypes are also evident in the parental strains. For example, the C57BL/6J strain exhibits lower levels of anxiety-related and fewer stress-related effects compared with the DBA/2J strain that 
exhibits greater fear-related responses [8-12]. Therefore, the BXD RI strains provide a powerful tool to study the genetic basis of anxiety- and fear-related behaviors in mice [13-16].

Single-trait QTL mapping methods are readily expandable to multiple-trait analysis. Jiang and Zeng [17] previously proposed a multivariate statistical model to conduct multiple-trait analysis. However, the same trait measured in different environments should be considered as distinct traits, and the maximum likelihood method applied in that approach increases the computational complexity. Although principal component and canonical variables have been used in multiple-trait analysis [18,19], the estimates are still incapable of delineating the effect(s) of each QTL. We previously proposed a full-QTL model for modeling the genetic architecture of a complex trait from multi-environment trials, using a mixed linear model approach [20]. By extending this statistical model, here, we performed multivariate QTL mapping of anxiety-related phenotypes under different conditions in an attempt to analyze main QTL effects, epistatic effects, as well as their interaction effects with different treatments. Simultaneously, a separate QTL mapping analysis was carried out for each measurement using a full QTL model based on mixed linear model. When these two mapping results were compared, we found some pleiotropic main and epistatic QTLs, several of which were sensitive to different treatment conditions.

\section{Materials and methods}

\subsection{Mouse strains}

In this study, we analyzed anxiety-related behavior in the open quadrants of an elevated zero maze, a standard tool for testing anxiety [21]. Seventy-one mice of the BXD RI strains, 60 to 120 -d-old were used in this study. This strain was derived by crossing the C57BL/6J (B6) and DBA/2J (D2) strains to produce the BXD1 through BXD32 strains (26 strains) [22] in the 1970s, and the BXD33 through BXD42 strains (9 strains) [23] in the 1990s. Genotypes of the BXD strains were generated at the University of Tennessee Health Science Center. A total of 3795 markers were genotyped, including 3033 SNPs and 762 SSRs. Many adjacent markers have identical strain distribution patterns; therefore, we selected 2320 markers for the subsequent analysis (1814 SNPs and 506 SSRs). To reduce computation complexity, only the 506 SSRs were used to map causal QTLs.

\subsection{Anxiety assay}

Anxiety-related behavior was examined in animals under five conditions: one, animals that were acutely restrained and received ethanol; two, animals that were acutely restrained and received saline; three, animals that received only a saline injection; four, animals that received only an ethanol injection; and five, animals that were not restrained and did not receive any injection. Acutely restrained animals were placed in an immobilization tube for $15 \mathrm{~min}$. Animals receiving injections received either ethanol (1.8 $\mathrm{g} / \mathrm{kg}$ ) or saline, and were returned to home cages. Three variables were recorded during the test session: Open-5, time spent in the open quadrants during the first $5 \mathrm{~min}$ of the session; Open +5 , time spent in the open during the last 5 min of the session; and Open10, time spent in the open during the entire test session.

Separate QTL mapping analyses were performed for each measurement (Open-5, Open+5, and Open10) to identify significant main and epistatic QTLs. Because any genetic relationships among these three behavioral measures are not taken into account in single-trait mapping computations, we also performed joint mapping of the QTL in an effort to determine pleiotropic effects, or common genetic influence on multiple traits. The two analyses (single and joint mapping) were carried out in QTLNetwork, a software package (http://ibi.zju.edu.cn/BCL/softwareNetwork_GPU. html) that can detect individual and epistatic interacting QTLs for individual and multiple traits [20]. In both analyses, the five experimental conditions were considered as different environments. Because the univariate mapping approach has already been described in detail by Yang et al. [20], and the multivariate mapping method will soon be published elsewhere, here we only briefly introduce the multivariate mapping approach.

\subsection{Statistical model for the multivariate mapping ap- proach}

Individual and epistatic interacting QTLs were detected using the following statistical model including general QTL effects and QTL by environment interaction effects,

$$
\begin{aligned}
y_{i j k}= & \mu_{i}+\sum_{l} a_{i l} x_{l j}+\sum_{l<h} a a_{i l h} x_{l j} x_{h j}+e_{k}+\sum_{l} \sum_{k} a e_{i l k} u_{l j} \\
& +\sum_{l<h} \sum_{k} a a e_{i l h k} u_{l j} u_{h j}+\varepsilon_{i j k},
\end{aligned}
$$

where, for the $i$ th trait $(i \in[1, m], m$ is the total number of traits), $y_{i j k}$ is the phenotypic value of the $j$ th line in the $k$ th environment; $\mu_{i}$ is the population mean of the $i$ th trait; $a_{i l}$ is the additive effect of the $l$ th QTL $\left(Q_{l}\right)$, fixed effect; $x_{l j}$ is the coefficient of the parameter $a_{i l} ; a a_{i l h}$ is the additive by additive effect between $Q_{l}$ and $Q_{h}$, fixed effect; $e_{k}$ is the random effect of the $k$ th environment; $a e_{i l k}$ and $a a e_{i l h k}$ are the additive by environment and additive $x$ additive by environment interaction effect, respectively, and are both random effects; and $\varepsilon_{i j k}$ is the random residual effect.

For the $i$ th trait, the statistical model in matrix notation is $\mathbf{y}_{i}=\mathbf{1} \mu_{i}+\mathbf{X} \mathbf{b}_{A i}+\mathbf{X} \mathbf{b}_{A A i}+\mathbf{I} \mathbf{e}_{E i}+\mathbf{U}_{A E_{i}} \mathbf{e}_{A E i}+\mathbf{U}_{A A E_{i}} \mathbf{e}_{A A E i}+\mathbf{I} \mathbf{e}_{\varepsilon i}$. 
For multiple traits, the multivariate model in matrix notation is

$$
\begin{aligned}
\mathbf{Y} & =\mathbf{1}_{n} \mathbf{b}_{\mu}^{T}+\mathbf{X}_{A} \mathbf{B}_{A}+\mathbf{X}_{A A} \mathbf{B}_{A A}+\mathbf{U}_{E} \mathbf{E}_{E}+\mathbf{U}_{A E} \mathbf{E}_{A E}+\mathbf{U}_{A A E} \mathbf{E}_{A A E}+\mathbf{E}_{\varepsilon} \\
& =\mathbf{X B}+\sum_{u=1}^{r+1} \mathbf{U}_{u} \mathbf{E}_{u},
\end{aligned}
$$

where $\mathbf{Y}$ is a $(n \times m)$ matrix of the phenotypic value $y_{i j k} ; \mathbf{b}_{\mu}$ is a $(m \times 1)$ column vector of the population mean $\mu_{i} ; \mathbf{B}_{A}$ is a $(s \times m)$ matrix of the additive effect $a_{i l} ; \mathbf{B}_{A A}$ is a $(t \times m)$ matrix of the additive by additive effect $a a_{i l h} ; \mathbf{X}_{A}$ and $\mathbf{X}_{A A}$ are the coefficient matrix of $\mathbf{B}_{A}$ and $\mathbf{B}_{A A}$, respectively; $\mathbf{E}_{E}$ is a $(p \times m)$ matrix of environmental effect $e_{k} ; \mathbf{E}_{A E}$ and $\mathbf{E}_{A A E}$ are the random matrix of $a e_{i l k}$ and $a a e_{i l h k}$, respectively; and $\mathbf{E}_{\varepsilon}$ is a $(n \times m)$ matrix of the random residual effect $\varepsilon_{i j k}$.

The mapping strategy adopted was similar to the fullQTL model proposed by Yang et al. [20]. It is divided into two steps: one, a 1D genome scan to map individual QTLs; and two, a 2D genome scan to detect all possible epistatic interactions. In the mapping approach, we used the Wilks' Lambda test instead of the $F$-test to determine the existence of QTLs because of the relationship among the multivariables.

\section{Results}

To explore the relationships among the three behavioral measures, we carried out correlational analyses for Open-5, Open +5 and Open10. The results showed that there were highly significant correlations among the phenotypes $\left(r_{P} \approx 0.78-0.94\right)$ and among the genotypes $\left(r_{G} \approx 0.82-0.96\right)$, suggesting that common or shared mechanisms may regulate the three related behavioral traits.

To identify the QTLs underlying these three behavioral measures, we mapped the QTLs for the individual QTL effect and for the epistatic effect of two QTLs, using both the univariate mapping (UVM) and multivariate mapping (MVM) approaches. The positions and additive effects of QTLs estimated by the UVM and MVM analyses are listed in Table 1.

As shown in Table 1 and Figure 1, two additive QTLs on chromosome 15 for Open-5 (Q15-19 at $46.1 \mathrm{cM})$ and Open10 (Q15-20 at 47.1 cM) were detected by UVM analysis. The close proximity of these QTLs suggests that the loci associated with these QTLs are closely linked. Interestingly, MVM analysis detected a single QTL (Q15-19) on chromosome 15 at $46.1 \mathrm{cM}$ associated with all three behavioral measures. In addition, three QTLs, in close proximity, on chromosome 13 at 28.4, 36.4, and $34.4 \mathrm{cM}$ were identified by UVM in Open-5, Open+5 and Open10 respectively. MVM analysis confirmed only one QTL (Q13-9) at 31.4 $\mathrm{cM}$ on chromosome 13. Because we obtained three separate but closely linked QTLs on chromosome 13 in the univariate analysis, and a single QTL when joint mapping was carried out, we suspected that there was actually a single QTL with pleiotropic effects on the behavioral traits. Again, when MVM and UVM were used to analyze the epistasis QTL, we found that MVM could detect pleiotropic effect from linked QTLs (Table 2). Together, these results indicated that MVM based on a mixed linear model approach could distinguish pleiotropic QTLs from closely linked QTLs.

Our findings show that the MVM approach has greater power in detecting QTLs than UVM analysis (Tables 1 and 2). UVM analysis detected a single QTL, Q5-53 for Open10 $\left(a \hat{=}-1.85, P<0.001, a e_{4} \hat{=} 1.65, P<0.05\right)$, however, MVM analysis revealed three different QTLs associated with each of the three behavioral measures (Open10 $a \hat{=}-0.90, P<$ $0.05, a e_{4} \hat{=} 1.63, P<0.05 ;$ Open $+5 a \hat{=}-1.15, P<0.01$;

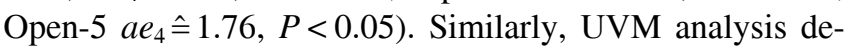
tected a single QTL, Q13-20, associated with Open10,

\begin{tabular}{|c|c|c|c|c|c|c|}
\hline \multirow{2}{*}{ Method } & \multicolumn{3}{|c|}{ QTL (position in cM) } & \multicolumn{3}{|c|}{ Additive effect (A) } \\
\hline & Open-5 & Open +5 & Open10 & Open-5 & Open +5 & Open10 \\
\hline \multirow{4}{*}{ UVM } & & & Q5-53 (91.3) & & & $-1.85^{\ddagger}$ \\
\hline & Q13-9 (28.4) & Q13-9 (36.4) & Q13-9 (34.4) & $-2.53^{\ddagger}$ & $-2.39^{\ddagger}$ & $-3.06^{+}$ \\
\hline & & & Q13-20 (64.3) & & & $-1.90^{\ddagger}$ \\
\hline & Q15-19 (46.1) & & Q15-20 (47.1) & $-4.21^{\ddagger}$ & & $-3.56^{\ddagger}$ \\
\hline \multirow{5}{*}{ MVM } & & & Q4-38 (74.3) & & & \\
\hline & Q5-53 (91.3) & Q5-53 (91.3) & Q5-53 (91.3) & & $-1.15^{* *}$ & $-0.90^{*}$ \\
\hline & Q13-9 (31.4) & Q13-9 (31.4) & Q13-9 (31.4) & $-3.32^{\ddagger}$ & $-3.76^{\ddagger}$ & $-3.54^{\ddagger}$ \\
\hline & Q13-20 (64.3) & & Q13-20 (64.3) & $-1.73^{\ddagger}$ & & $-1.13^{+}$ \\
\hline & Q15-19 (46.1) & Q15-19 (46.1) & Q15-19 (46.1) & $-3.10^{\ddagger}$ & $-1.45^{\ddagger}$ & -2.28 \\
\hline
\end{tabular}

Table 1 QTL positions and additive effects obtained by UVM and MVM analyses ${ }^{\text {a) }}$

a) UVM, univariate mapping; MVM, multivariate mapping. Q $m-c$ is the QTL located at the left side of marker $m$ on chromosome $c$. $* * *,+$ and $\ddagger$ represent $0.05,0.01,0.005$ and 0.001 significant levels ( $P$ values), respectively. The empty cells in the additive effect column indicated that the additive effect is insignificant. 


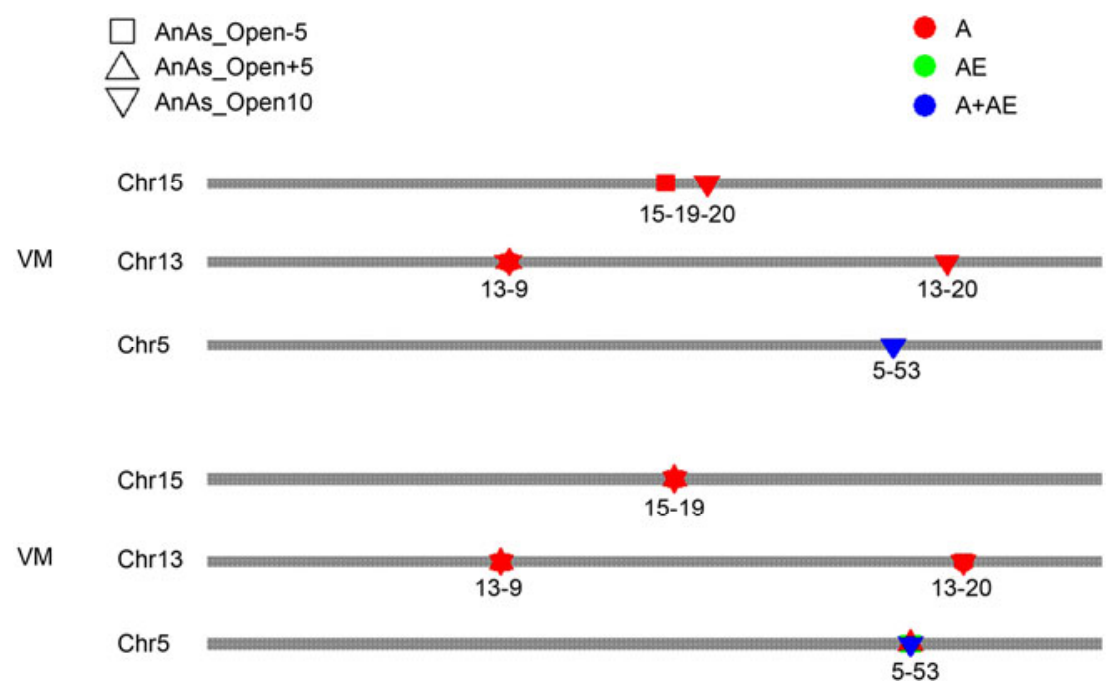

Figure 1 Positions of the main QTLs detected by separate and joint QTL mapping. UVM, univariate mapping (individual trait mapping); MVM, multivariate mapping (joint-trait mapping). AnAs_Open-5, AnAs_Open+5 and AnAs_Open10 refer to the three mice anxiety assay measures: testing for the first 5 $\mathrm{min}$, testing for the last $5 \mathrm{~min}$, and testing the whole $10 \mathrm{~min}$. Red represents a QTL with only additive effects; green represents a QTL with additive $\times$ treatment interaction effects (AE); and blue represents a QTL with both additive and additive $\times$ treatment interaction effects $(\mathrm{A}+\mathrm{AE})$.

Table 2 Positions and epistatic effects of two QTLs obtained by UVM and MVM analyses ${ }^{\text {a) }}$

\begin{tabular}{|c|c|c|c|c|c|c|}
\hline \multirow{2}{*}{ Method } & \multicolumn{3}{|c|}{ QTL×QTL (position in cM) } & \multicolumn{3}{|c|}{ Additive $\times$ Additive effect (AA) } \\
\hline & Open-5 & Open+5 & Open10 & Open-5 & Open +5 & Open10 \\
\hline \multirow{2}{*}{ UVM } & & Q7-33×Q14-7 (77.6/21.8) & & & $3.94^{\ddagger}$ & \\
\hline & Q9-9×Q17-19 (26.1/52.5) & Q9-8×Q17-18 (24.5/50.9) & & $3.62^{\ddagger}$ & $3.21^{\ddagger}$ & \\
\hline \multirow{3}{*}{ MVM } & Q7-33×Q14-7 (77.6/21.8) & Q7-33×Q14-7 (77.6/21.8) & Q7-33×Q14-7 (77.6/21.8) & $3.03^{\ddagger}$ & $3.56^{\ddagger}$ & $3.29^{\ddagger}$ \\
\hline & Q7-33×Q14-14 (77.6/29.4) & Q7-33×Q14-14 (77.6/29.4) & Q7-33×Q14-14 (77.6/29.4) & & $1.11^{*}$ & \\
\hline & Q9-10×Q17-20 (33.7/54.7) & Q9-10×Q17-20 (33.7/54.7) & Q9-10×Q17-20 (33.7/54.7) & $3.28^{\ddagger}$ & $3.17^{\ddagger}$ & $3.22^{\ddagger}$ \\
\hline
\end{tabular}

a) UVM, univariate mapping; MVM, multivariate mapping. Q $m-c$ is the QTL located at the left side of marker $m$ on chromosome $c$. $\$$ represents 0.001 significant levels ( $P$ values). The empty cells in the additive $\times$ additive effect column indicated that the epistatic effect is insignificant.

while MVM analysis detected the same Q13-20 associated with both the Open10 and Open-5 measures. Similarly, UVM analysis only detected an interaction between Q7-33 and Q14-7 for Open+5 $(a a \hat{=} 3.94, P<0.001)$, but MVM analysis estimated that these QTLs were associated with all three measures (Open-5 aa $\hat{=} 3.03, P<0.001$; Open +5 $a a \hat{=} 3.56, P<0.001$; Open10 $a a \hat{=} 3.29, P<0.001)$. Finally, the interaction between Q9-10 and Q17-20 was detected only when MVM analysis was performed (Open-5 $a_{a} e_{4} \hat{=}$ -2.96, $P<0.005$; Open+5 $a a \hat{=} 1.11, P<0.05$; Open 10 $\left.a a e_{4} \hat{=}-1.69, P<0.05\right)$ and not with UVM.

To look for potential candidate genes within the QTL intervals, we listed all the known genes which underly the ten most significant QTLs and are reported to be related to anxiety. We identified ten potential candidate genes: one, Tmem132d, in the Q5-53 interval; four, Dtnbpl, Drdla, Cplx2, and Grk6, in the Q13-9 QTL interval; two, Pik3rl and Htrla, in the Q13-20 QTL interval; and three, Arc, Apol3, and Hsfl, in the Q15-19 interval. Interestingly, two of these genes (Dtnbpl and Drdla) are known to be associ- ated with dopamine systems and, among other things, dopamine is known to act upon receptors in the brain that can alter the way the brain reacts to normal social situations, leading to anxiety. Previous studies have indicated that Dtnbp1 is a risk gene and a potential genetic basis for schizophrenia and affective disorders such as bipolar or major depressive disorder (MDD) [24-26]. Similarly, a Drd1a polymorphism has been associated with the presence of psychosis and aggressive behavior in patients with Alzheimer disease (AD) [27-29].

\section{Discussion}

In most biological experiments, multiple phenotypes are observed and measured simultaneously in the same individuals. Such phenotypes are usually highly correlated with one another and may be under the control of common genetic mechanisms. As described above, two different approaches can be used to examine pleiotropic QTLs, either 
by mapping each trait separately (UVM) or by joint analysis of traits (MVM). Because the MVM approach takes into account covariance in the traits studied, it has four distinct advantages over the UVM approach. The MVM approach: (1) increases the statistical power of QTL detection; (2) improves the precision of estimation for QTL intervals; (3) allows for hypothesis testing involving relationships among the multiple traits, and (4) detects pleiotropic QTLs [17,30-33]. In addition, the MVM approach can detect additive and epistatic QTLs with significant genetic effects which generally are not detected with UVM methods.

Although the MVM approach offers several advantages over the UVM approach, there is a limitation that should be considered. The MVM approach may identify what appears to be a single pleiotropic QTL, but if this approach alone is used, it can be difficult to determine whether or not the QTL is actually a single QTL or two QTLs in very close proximity to one another. This problem, however, can be resolved by separately testing specific genomic regions within the region identified by joint analysis to distinguish between separate and pleiotropic QTLs [17].

C57BL/6J (abbreviated B6) mice showed lesser anxietyand stress-related behaviors than strain DBA/2J (abbreviated D2) mice [8-12] and the reported continuously distributed observations of the BXD lines were assumed to be driven by interactions between multiple genes. In this study, we used the MVM mapping approach to detect QTLs involved in anxiety-related behaviors in the mouse. This approach proved to be an accurate and powerful tool to detect pleiotropic additive and epistatic interacting QTLs. The MVM approach is useful in identifying candidate genes involved in multiple anxiety-related traits. The five individual QTLs and three epistatic interactions detected by MVM approach were found to have similar positions and effects. Based on these estimated QTLs, we investigated the genetic basis of the observed anxiety-like behavior in the mice. The individual QTL Q5-53 seemed to have a negative additive effect in two of the test measures (Open+5 $a \hat{=}-1.15, P<0.01$; Open10 $a \hat{=}-0.90, P<0.05)$, suggesting that Q5-53 may be associated with enhanced fear or anxiety. However, we noted that alcohol may reduce the anxiety by a small amount, because QTL Q5-53 exhibited a significant positive additive by environment effects when the mice were acutely restrained and received ethanol. Similarly, the effects of the epistatic interactions allowed us to deduce how the identified QTLs might be associated with the fearlike behaviors. For instance, the interaction between QTLs Q9-10 and Q17-20 showed positive additive $\times$ additive effects for the three behavioral measures (Open-5 $a a \hat{=} 3.28$, $P<0.001$; Open+5 $a a \hat{=} 3.17, P<0.001$; Open10 $a a \hat{=} 3.22$, $P<0.001$ ), indicating that their interaction may moderate the anxiety response. Furthermore, we investigated these QTLs that were found to significantly modulate the anxiety or fear response, and identified candidate casual genes located in the significant support interval which is defined by
$F$-value drop. A recent report identified Tmem132d as a candidate gene for anxiety-related behavior [34]. This gene, located in the region of Q5-53, has been reported to be significantly associated with panic disorder in humans. Besides, the mouse experiments suggest Tmem132d is an important gene for anxiety-related behavior. Previous studies have revealed that Dtnbpl [35], located in the support interval of Q13-9, is a candidate gene for risk of schizophrenia, by causing depression and anxiety.

In the present study, ten significant QTLs detected by MVM have been shown to be potential casual loci for the anxiety-related response. We concluded that, compared with UVM, the MVM approach was more powerful in uncovering the genetic and genomic basis of multiple co-related complex traits.

This work was supported by National Basic Research Program of China (2011CB109306), National Key Technologies R\&D Program in China (2009ZX08009-004B), CNTC (110200701023), and YNTC (08A05).

1 Phillips T J, Belknap J K. Complex-trait genetics: Emergence of multivariate strategies. Nat Rev Neurosci, 2002, 3: 478-485

2 Levene $H$. Genetic equilibrium when more than one ecological niche is available. Am Nat, 1953, 87: 331-333

3 Gillespie J H, Turelli M. Genotype-environment interactions and the maintenance of polygenic variation. Genetics, 1989, 121: 129-138

4 Mackay T F C, Stone E A, Ayroles J F. The genetics of quantitative traits: Challenges and prospects. Nat Rev Genet, 2009, 10: 565-577

5 Caspi A, Moffitt T E. Opinion-gene-environment interactions in psychiatry: Joining forces with neuroscience. Nat Rev Neurosci, 2006, 7: 583-590

6 Hariri A R, Holmes A. Genetics of emotional regulation: The role of the serotonin transporter in neural function. Trends Cogn Sci, 2006, 10: 182-191

7 Uher R, McGuffin P. The moderation by the serotonin transporter gene of environmental adversity in the aetiology of mental illness: Review and methodological analysis. Mol Psychiatr, 2008, 13: 131-146

8 Brigman J L, Mathur P, Lu L, et al. Genetic relationship between anxiety-related and fear-related behaviors in BXD recombinant inbred mice. Behav Pharmacol, 2009, 20: 204-209

9 Belzung C, Griebel G. Measuring normal and pathological anxietylike behaviour in mice: A review. Behav Brain Res, 2001, 125: 141149

10 Jacobson L H, Cryan J F. Feeling strained? Influence of genetic background on depression-related behavior in mice: A review. Behav Genet, 2007, 37: 171-213

11 Millstein R A, Holmes A. Effects of repeated maternal separation on anxiety- and depression-related phenotypes in different mouse strains. Neurosci Biobehav R, 2007, 31: 3-17

12 Tannenbaum B, Anisman H. Impact of chronic intermittent challenges in stressor-susceptible and resilient strains of mice. Biol Psychiat, 2003, 53: 292-303

13 Caldarone B, Saavedra C, Tartaglia K, et al. Quantitative trait loci analysis affecting contextual conditioning in mice. Nat Genet, 1997, 17: 335-337

14 Owen E H, Christensen S C, Paylor R, et al. Identification of quantitative trait loci involved in contextual and auditory-cued fear conditioning in BXD recombinant inbred strains. Behav Neurosci, 1997, 111: 292-300

15 Radcliffe R A, Lowe M V, Wehner J M. Confirmation of contextual fear conditioning QTLs by short-term selection. Behav Genet, 2000, 30: 183-191

16 Wehner J M, Radcliffe R A, Rosmann S T, et al. Quantitative trait 
locus analysis of contextual fear conditioning in mice. Nat Genet, 1997, 17: 331-334

17 Jiang C J, Zeng Z B. Multiple-trait analysis of genetic-mapping for quantitative trait loci. Genetics, 1995, 140: 1111-1127

18 Mangin B, Thoquet P, Grimsley N. Pleiotropic QTL analysis. Biometrics, 1998, 54: 88-99

19 Weller J I, Wiggans G R, VanRaden P M, et al. Application of a canonical transformation to detection of quantitative trait loci with the aid of genetic markers in a multi-trait experiment. Theor Appl Genet, 1996, 92: 998-1002

20 Yang J, Zhu J, Williams R W. Mapping the genetic architecture of complex traits in experimental populations. Bioinformatics, 2007, 23: $1527-1536$

21 Shepherd J K, Grewal S S, Fletcher A, et al. Behavioral and pharmacological characterization of the elevated zero-maze as an animalmodel of anxiety. Psychopharmacology, 1994, 116: 56-64

22 Taylor B A. Recombinant inbred strains. In: Lyon M L, Searle A G, eds. Genetic Variation in the Laboratory Mouse. 2nd ed. Oxford: Oxford University Press, 1989

23 Taylor B A, Wnek C, Kotlus B S, et al. Genotyping new BXD recombinant inbred mouse strains and comparison of BXD and consensus maps. Mamm Genome, 1999, 10: 335-348

24 Feng Y Q, Zhou Z Y, He X, et al. Dysbindin deficiency in sandy mice causes reduction of snapin and displays behaviors related to schizophrenia. Schizophr Res, 2008, 106: 218-228

25 Voisey J, Swagell C D, Hughes I P, et al. A polymorphism in the dysbindin gene (DTNBP1) associated with multiple psychiatric disorders including schizophrenia. Behav Brain Funct, 2010, 6: 41

26 Domschke K, Lawford B, Young R, et al. Dysbindin (DTNBP1)-a role in psychotic depression? J Psychiatr Res, 2011, 45: 588-595
27 Sweet R A, Nimgaonkar V L, Kamboh M I, et al. Dopamine receptor genetic variation, psychosis, and aggression in Alzheimer disease. Arch Neurol, 1998, 55: 1335-1340

28 Holmes C, Smith H, Ganderton R, et al. Psychosis and aggression in Alzheimer's disease: The effect of dopamine receptor gene variation. J Neurol Neurosurg Psychiatry, 2001, 71: 777-779

29 Ni X Q, Trakalo J M, Mundo E, et al. Linkage disequilibrium between dopamine D1 receptor gene (DRD1) and bipolar disorder. Biol Psychiat, 2002, 52: 1144-1150

30 Williams J T, Van Eerdewegh P, Almasy L, et al. Joint multipoint linkage analysis of multivariate qualitative and quantitative traits. I. Likelihood formulation and simulation results. Am J Hum Genet, 1999, 65: 1134-1147

31 Williams J T, Begleiter H, Porjesz B, et al. Joint multipoint linkage analysis of multivariate qualitative and quantitative traits. II. Alcoholism and event-related potentials. Am J Hum Genet, 1999, 65: $1148-1160$

32 Korol A B, Ronin Y I, Itskovich A M, et al. Enhanced efficiency of quantitative trait loci mapping analysis based on multivariate complexes of quantitative traits. Genetics, 2001, 157: 1789-1803

33 Henshall J M, Goddard M E. Multiple-trait mapping of quantitative trait loci after selective genotyping using logistic regression. Genetics, 1999, 151: 885-894

34 Erhardt A, Czibere L, Roeske D, et al. TMEM132D, a new candidate for anxiety phenotypes: Evidence from human and mouse studies. Mol Psychiatry, 2011, 16: 647-663

35 Wray N R, James M R, Handoko H Y, et al. Association study of candidate variants from brain-derived neurotrophic factor and dystrobrevin-binding protein 1 with neuroticism, anxiety, and depression. Psychiatr Genet, 2008, 18: 219-225

Open Access This article is distributed under the terms of the Creative Commons Attribution License which permits any use, distribution, and reproduction in any medium, provided the original author(s) and source are credited. 